\title{
Isolated Caudate Lobectomy: Glissonean Pedicle Transection Method, and Combined Right-left Side Approach. A Case Series Report
}

Luong Hiep ( $\sim$ Hiep1995hsgs@gmail.com )

\section{Case report}

Keywords: Caudate lobe, Glissonean pedicle, liver tumor

Posted Date: September 16th, 2021

DOI: https://doi.org/10.21203/rs.3.rs-907829/v1

License: (c) (i) This work is licensed under a Creative Commons Attribution 4.0 International License.

Read Full License 


\section{Abstract}

Tumors located in caudate lobe may be primary tumors, or metastases from other sites. Isolated caudate lobectomy (ICL) is a challenging procedure due to its complex structure and location. The access route to caudatelobe has an important role in the success of the operation. A combined right and left side approach is recommended for benign or suspected malignant tumor located anterior to the vena cava or entirely in the caudate lobe, which radical resection of the caudate is necessary. We report two cases of successfully with Glissonean pedicle transection method described by Takasaki and combined right and left side approach. We found this to be a safe and effective approach, which can be applied to all case of benign tumors; or in the case of malignant tumors located entirely in the caudate lobe when extended hepatic resection is not possible due to poor liver function. or small remnant liver volume.

\section{Highlights}

- Caudate lobe is the part of the liver locate deep in the liver, caranial to the hilar plate, behind three main hepatic veins, and in front of the inferior vena cava (IVC).

- Isolated complete caudate lobectomy (CCL) is a challenging technique procedure due to its complex structure and location.

- Our technique consists of three main steps: Isolation of caudate lobe from the hepatic pedicles, the 3 main hepatic veins, and finally, the IVC.

- A combined right and left approach with glissonean pedicle transection method is recommended for tumors located entirely in the caudate lobe.

\section{Instructions}

Caudate lobe is the part of the liver that located cranial to the hilar plate, behind three hepatic veins, and in front of the inferior vena cava (IVC). According to Kumon classification, the caudate lobe is divided into three regions: Spigel lobe, the process portion or the caudate process, and the paracaval portion ${ }^{1}$. Tumors of caudate lobe may be a primary tumor in the liver, or a tumor from another site that has metastasized. Because of its anatomical location strongly related to the hilar plate consist of Glisson pedicles, the hepatic vein anteriorly, and the short hepatic vein drains directly from the caudate lobe to the IVC and is posteriorly related to the anterior aspect of the vena cava, therefore, complete caudate lobectomy (an anatomic resection of segment I and segment IX resection with Couinaud's classification ${ }^{2}$ ) is a huge challenge for the surgeons. Isolated complete caudate lobe resection can be performed alone or in combination with right lobectomy, right and left hepatectomy. Isolated complete caudate lobe resection is indicated for benign tumors located in all parts of this liver region. For hepatic malignancy, especially in patients with hepatocellular carcinoma, often associated with cirrhosis, individual caudate lobectomy is proposed for tumors localized in this region to preserve remaining liver function. 
Generally, approach of caudate lobe has four ways including: right, left side; right and left coordination; and anteriorly through the parenchymal cut of the liver. Depending on the location, characteristics of the

tumor and the type of caudate lobectomy, the surgeon will have a suitable approach to the tumor ${ }^{3-6}$. For complete caudate lobe resection, we found that the right and left two-way approach combined with Glissonean pedicle control by Takasaki's technique is an easy and safe method. Herein we report two cases of successfully with Glissonean pedicle isolation using Takasaki's technique and right-left approach. All our work has been reported in line with the SCARE criteria and guidelines ${ }^{7}$.

\section{Surgical Procedure}

Based on the characteristics of the segment I location, which is the part of the liver located in front of the vena cava, below the hepatic veins, and cranial to the hilar plate, our approach aims to isolate the entire caudate lobe from these anatomical structures following steps:

- First, we dissected the caudate lobe from the hilar plate. We performed glissonean pedicle transection method (described by professor Takasaki) this step: cholecystectomy, dissection of cystic plate and umbilical plate (thickened parts of the

- Glissonean pedicle comprising of collagen-rich connective tissues, which was first described by Couinaud ${ }^{8}$ ) and resection of the anchors, which are the thin, cord-like structures located at the orifices of the Glissonean pedicles that connect with Laennec's capsule ${ }^{8}$ ). Then, we could isolate and tape the right and left hepatic pedicles and maximally move these pedicles (Fig. 4). We dissected clearly the right caudate lobe Glissonean pedicle, which coming from the right Glissonean pedicle (usually about $1 \mathrm{~cm}$ from the posterior segment pedicle). Coming to the left-side, after we opened of the lesser omentum, segment I is blunt dissected posteriorly to the ligamentum venosum to show its left pedicles. There are usually two pedicles enter segment I from the left Glissonean pedicle.

- Isolating of the caudate lobe from the IVC: We transected both side coronary and triangular ligaments to fully mobilize the right and left liver. We ligated all the short hepatic veins of the caudate lobe, which directly drains into the IVC in both right and left side (Fig. 1). From the left side, we lifted the Spigel lobe and ligated all the veins all the way up to the left hepatic vein. From the right side, we ligated the inferior right hepatic veins, divided the hepatocaval ligament, tie all the short hepatic veins from the caudate process, until the right and left sides are connected.

- Isolating of caudate lobe from the hepatic veins: We did this step along with parenchymal resection. We also approach from both sides, and an upward path to transect the caudate process. The procedure usually starts from the left side, the liver parenchyma is resected just below the ligamentum venosum, we transected the liver parenchyma from the inferior surface of the middle and left hepatic veins (Fig. 2). At the bottom, we taped the right Glissonean pedicle, cut the caudate process and separated it from the right liver parenchyma. This transection line will meet the 
transection from the left side. We used an inferior approach to transect the parenchymal of the lower right quadrant of caudate lobe. Then, we approached to the right side: We opened the parenchyma from this side will pass up from the right hepatic vein root and down to meet the caudal process section made from below (Fig. 3). It is important to note that this parenchymal opening should be directed toward parenchymal resection adjacent to the inferior surface of the right hepatic vein. When transection line found the landmark of the right hepatic vein, then we continued until we met the left transection line and removed the entire caudate lobe. During parenchymal transection, we applied selective right and left pedicle clamps when approached from each side, respectively.

- Final stage: we carefully performed hemostasis of liver resected surface (Fig. 5). We also placed a cystic duct catheter to check for bile leaks.

\section{Results}

The above technique was successfully performed on 2 following patients:

The first case was a 63-year-old female patient with a history of chronic hepatitis B without any treatment. She was diagnosed with liver tumor because of abdominal pain. Computed tomography (CT) result showed a tumor occupying nearly the entire caudate lobe with characteristics of HCC, $46 \mathrm{~mm}$ in diameter, compressed inferior vena cava, close to the hilar plate, right and left hepatic veins but not invasive, no thrombus portal vein (Fig. 6). The serum AFP $>400 \mathrm{ng} / \mathrm{mL}$. Liver function tests were within normal limit. During the resection of the liver parenchyma, we injured the middle hepatic vein. We managed by lifting the tapes over the common trunk of the left and middle hepatic veins, with temporary Pringle maneuver technique and sutured with Prolene 5/0. The operation time was 130 minutes, the amount of blood lost during the surgery was about $250 \mathrm{ml}$. We tested bile leakage by Injecting normal saline through the cystic duct via a catheter. The patient underwent postoperative course uneventfully. We discharged the patient at postoperative day 7 . The pathological result was moderately differentiated hepatocellular carcinoma occupies nearly the entire caudate lobe. The resection margin was less than 0.5 $\mathrm{cm}$, and negative. Follow-up 9 months after surgery, there is no recurrence on CT and magnetic resonance imaging (MRI).

The second case was a 39-year-old female patient without any medical history. She was under routine check-up, and a liver tumor was detected. The serum AFP level was $3.03 \mathrm{ng} / \mathrm{mL}$; and HbsAg, HCV-Ab results were negative. The 4 phase-CT result showed a $45 \mathrm{~mm}$ lesion in caudate lobe, hypointense before injection, had washout characteristic after contrast injection, with irregular margins (Fig. 7). There was no portal vein thrombosis, no invasion of the hilar plate, IVC, and hepatic veins. Liver MRI showed a liver tumor about $50 \mathrm{~mm}$ in size also had washout characteristic, clear margin, close to the hepatic veins and IVC (Fig. 8). The Gastroscopy and colonoscopy results were normal. While we could not exclude malignancy, and biopsies are difficult due to tumor location, we decided to resect the entire caudate lobe according to described technique. Surgery time was 120 minutes, no complications during surgery, blood loss during surgery was about $150 \mathrm{ml}$. There was no bile leakage found. The patient also underwent uneventfully postoperative course and was discharged after 7 days of hospital stay. Pathological result 
was focal nodular hyperplasia. After 7 months of follow-up, the patient is now healthy and has normal daily activities.

\section{Discussion}

The caudate lobe, also known as segment I according to Kumon's classification, is the portion of the liver that lies between the vascular structures: the hilar plate, hepatic veins, and the IVC. Kumon divides the segment I into 3 parts: the Spigel lobe, also known as S1I, which lies below the lesser omentum and extends to the left posterior hepatic part of vena cava; the anterior venous part is the part located in front of the vena cava and to the right of the Spigel lobe, also known as S1r, which is closely associated with the right hepatic vein and the middle hepatic vein; the caudate process is the right portion of the anterior venous part also known as S1c ${ }^{1}$.

According to Takasaki, the liver was divided into 4 segments: the right segment, the middle segment, the left segment, and the caudal region. The caudal region occupies about $10 \%$ of liver volume and supplied by several small Glissonean pedicles directly from the first branch at the hepatic hilum. This is the region of the liver located in front of the vena cava, and its veins drain directly into the vena cava. However, this part of the liver is only adjacent but not attached to the vena cava. The boundaries of the three lobes of the liver with the caudal region are delimited by triangles as shown in the figure. The anterior triangle of the vena cava separates the left and caudal lobes, and the lateral triangle of the vena cava separates the caudal lobe from the right lobe 9 .

Our approach to the caudate lobe is based on these above two anatomical perspectives. In order to find and ligate the caudate lobe pedicles from the hilar plate, we detached the hilum plate, and isolate the right and left Glissonean pedicle. Then we could find and the ligate the pedicle to the caudate lobe, and also created more space for dissection in this hard-to-reached area. The lateral and anterior triangle segment of the vena cava in the figure? shows us the left and right liver demarcation to separate the caudate lobe from the right and left liver. When approacg to the left side, we can rely on an anatomical landmark is the ligamentum venosum. The liver parenchymal transection line must be close to this ligament to avoid injury to the middle and left hepatic vein. Kumon's Classification allows a 3-way parenchymal resection approach according to our technique from the left side (Spigel lobe), from below (the caudal process) and from the right side (the paracaval portion).

For a successful ICL, in addition to the knowledge of surgical anatomy, the way to approach liver tumor also plays a very important role. Depending on the location and pathology of tumor and the liver function, an appropriate approach and method should be selected to ensure the radical oncology and to limit complications, especially liver failure after an extended liver resection. Regarding the location for a rational approach, Hasegawa et al. ${ }^{10}$ classified liver tumors originating from the caudate lobe into 5 types: 
- Type 1: Lesions in the Spigel lobe's upper part

- Type 2: Lesions in the Spigel lobe's lower part

- Type 3: Lesions around the vena cava (paracaval portion)

- Type 4: Lesions in the caudate process

- Type 5: Diffuse lesions of the entire caudate lobe

Our first patient is a case of HCC, a tumor of type 5 according to Hasegawa's classification, although the tumor located close to the right hepatic vein, the caudate lobectomy with right hepatectomy could be more radical, but because of cirrhosis and the remaining liver volume was not enough. In the second case, the tumor completely occupied the anterior vena cava of the subsegment I and pushed to the Spigel lobe and could be classified between type 3 and 5 . After we removed the entire caudate lobe an frozen section result was a benign lesion. Thus, our choice of ICL in both cases is reasonable in different circumstances.

During isolated caudate lobectomy, there are four main approaches: left-sided approach, right-sided approach, anterior approach, and right and left combined approach. For tumors in the left region (Spigel lobe), the left approach is sufficient, but for large tumors or tumors in the anterior area of vena cava, it is often necessary to combine with the right approach or use the anterior parenchymal opening. Anterior parenchymal opening approach provides good visibility and access for subsegment I resection, especially for large tumors and closely related to the hepatic veins ${ }^{11,12}$ but it increases the risk of bleeding and prolongs the surgical time ${ }^{13,14}$. A combined left and right approach is recommended for most tumors of caudate lobe 4,15 , especially for tumors larger than $4 \mathrm{~cm}$ in diameter, which are of primary origin from the paracaval portion or in the entire caudate lobe, or those that are thought to be malignancies, require total caudate lobectomy to remove the tumor.

The exposure and ligation of the short hepatic veins is a difficult task in ICL ${ }^{4}$. Ligation and resection of the short hepatic veins release the entire caudate lobe from the IVC and facilitate management of the hepatic veins and control of hemostasis during parenchymal resection. We found that the process of controlling the short hepatic veins was not too difficult after fully mobilized the right and left liver, when combining from both the right and left sides together with fine dissection.

We found that we sould isolate and tape the hepatic veins before performing parenchymal transection because the caudate lobe parenchyma is in contact with the posterior surface of the hepatic veins. So, we could reduce the risk of injuring these veins. The transection of the ligamentum venosum will help control the left and middle hepatic veins easier, but we did not divide this ligament. We blunt dissected and pushed it from the liver for the purpose of using this ligament as a landmark to start transecting the liver parenchyma from the left side. 
Resection of liver parenchyma is the most challenging stage, we transected the liver parenchyma and had to ensures not to rupture the tumor, not to damage to the hepatic veins, especially the middle hepatic vein. Because the resection location deep, and close to the hepatic veins, it is easy to damage the hepatic veins. We found that, it is very difficult to control and manage the ịnured vein during this stage. Other authors have used many ways to safely and effectively cut the liver parenchyma such as using hanging maneuver procedure ${ }^{16}$, or using dye agents to define the hepatectomy demarcation ${ }^{3}$, or using anterior parenchyma resection provides better visibility, but anterior parenchymal resection increases operative time and increases the risk of bleeding ${ }^{13,14}$. To secure the parenchymal resection, we suggest the following tactics: first, after a three-pronged approach described above, detachment of the hilum plate and ligation of the pedicles of caudate lobe help to create space for dissection as well as recognize parenchymal ischemic area. The second is selective clamp the right and left glissonean pedicles, respectively when transection parenchyma from both sides and only did the Pringle maneuver if there was severe bleeding; and finally, we note the landmarks of parenchymal demarcation on both sides, on the left is just below the ligamentum venosum and on the right is from the origin following the inferior border of the right hepatic vein, to ensure the best transetion area, and avoid damaging the hepatic veins.

\section{Conclusions}

The key of success for hepatectomy is understanding the anatomy of the caudate lobe, choosing the suitable approach, and performing careful and meticulous dissection. The glissonean transection method to isolate the glissonean pedicle and using a combined right- and left-side approach is safe and effective for non-invasive and vessels-related tumor.

\section{Abbreviations}

ICL Isolated caudate lobectomy

CT Computed tomography

FNH Focal nodular hyperplasia

IVC Inferior vena cava

HCC Hepatocellular carcinoma

HV Hepatic vein

MRI Magnetic resonance imaging

PV Portal vein

\section{Declarations}




\section{Funding}

The authors declare no funding for this study.

\section{Conflict of interests}

The authors declare that they have no conflicts of interests.

\section{Consent to participate.}

The patients have consented to the submission of the case report for submission to the journal.

\section{Consent to publication}

Not applicable

\section{Availability of data and material}

Data is available upon reasonable request and with permission of Bach Mai Hospital.

\section{References}

1. Kumon M. Anatomical Study of the Caudate Lobe with Special Reference to Portal Venous and Biliary Branches Using Corrosion Liver Casts and Clinical Application. Liver cancer Feb. 2017;6(2):161-70. doi:10.1159/000454682.

2. Makuuchi M, Yamamoto J, Takayama T, et al. Extrahepatic division of the right hepatic vein in hepatectomy. Hepato-gastroenterology Apr. 1991;38(2):176-9.

3. Midorikawa Y, Takayama T. Caudate lobectomy (segmentectomy 1) (with video). Journal of hepatobiliary-pancreatic sciences. Jan. 2012;19(1):48-53. doi:10.1007/s00534-011-0450-1.

4. Chaib E, Ribeiro MA Jr, Silva Fde S, Saad WA, Cecconello I. Caudate lobectomy: tumor location, topographic classification, and technique using right- and left-sided approaches to the liver. American journal of surgery Aug. 2008;196(2):245-51. doi:10.1016/j.amjsurg.2007.11.020.

5. Sasada A, Ataka K, Tsuchiya K, Yamagishi H, Maeda H, Okada M. Complete caudate lobectomy: its definition, indications, and surgical approaches. HPB surgery: a world journal of hepatic pancreatic biliary surgery. 1998;11(2):87-93. doi:10.1155/1998/92312. discussion $93-5$.

6. Chaib E, Ribeiro MAF Jr, YEDMd S, D'Albuquerque LAC. Anterior hepatic transection for caudate lobectomy. Clinics. 2009;64(11):1121-5. doi:10.1590/S1807-59322009001100013.

7. Agha RA, Franchi T, Sohrabi C, Mathew G, Kerwan A. The SCARE 2020 Guideline: Updating Consensus Surgical CAse REport (SCARE) Guidelines. International journal of surgery (London England) Dec. 2020;84:226-30. doi:10.1016/j.jjsu.2020.10.034.

8. Sugioka A, Kato Y, Tanahashi Y, et al. Standardization of Anatomic Liver Resection Based on Laennec's Capsule. Surgery, Gastroenterology and Oncology. 2020/04/30/ 2020;25(2):57. 
9. Yamamoto M, Katagiri S, Ariizumi S-i, Kotera Y, Takahashi Y. Glissonean pedicle transection method for liver surgery (with video). J Hepato-Biliary-Pancreat Sci. 2012;19(1):3-8. doi:10.1007/s00534011-0443-0.

10. Hasegawa H, de Cervens T, Yamasaki S, Kosuge T, Takayama T, Shimada K. [Surgical strategy for hepatocellular carcinoma of the caudate lobe]. Journal de chirurgie Dec. 1991;128(12):533-40. Stratégie chirurgicale pour les carcinomes hépatocellulaires du lobe caudé.

11. Dai WD, Huang JS, Hu JX. Isolated caudate lobe resection for huge hepatocellular carcinoma (10 cm or greater in diameter). The American surgeon Feb. 2014;80(2):159-65.

12. Yang JH, Gu J, Dong $P$, et al. Isolated complete caudate lobectomy for hepatic tumor of the anterior transhepatic approach: surgical approaches and perioperative outcomes. World journal of surgical oncology Aug 16. 2013;11:197. doi:10.1186/1477-7819-11-197.

13. Ahanatha Pillai S, Sathyanesan J, Perumal S, et al. Isolated caudate lobe resection: technical challenges. Annals of gastroenterology. 2013;26(2):150-5.

14. Sakamoto Y, Nara S, Hata S, et al. Prognosis of patients undergoing hepatectomy for solitary hepatocellular carcinoma originating in the caudate lobe. Surgery Nov. 2011;150(5):959-67. doi:10.1016/j.surg.2011.03.005.

15. Tian G, Chen Q, Guo Y, Teng M, Li J. Surgical strategy for isolated caudate lobectomy: experience with 16 cases. HPB surgery: a world journal of hepatic pancreatic biliary surgery. 2014;2014:983684-4. doi:10.1155/2014/983684.

16. López-Andújar R, Montalvá E, Bruna M, et al. Step-by-step isolated resection of segment 1 of the liver using the hanging maneuver. American journal of surgery Sep. 2009;198(3):e42-8. doi:10.1016/j.amjsurg.2009.02.012.

\section{Figures}




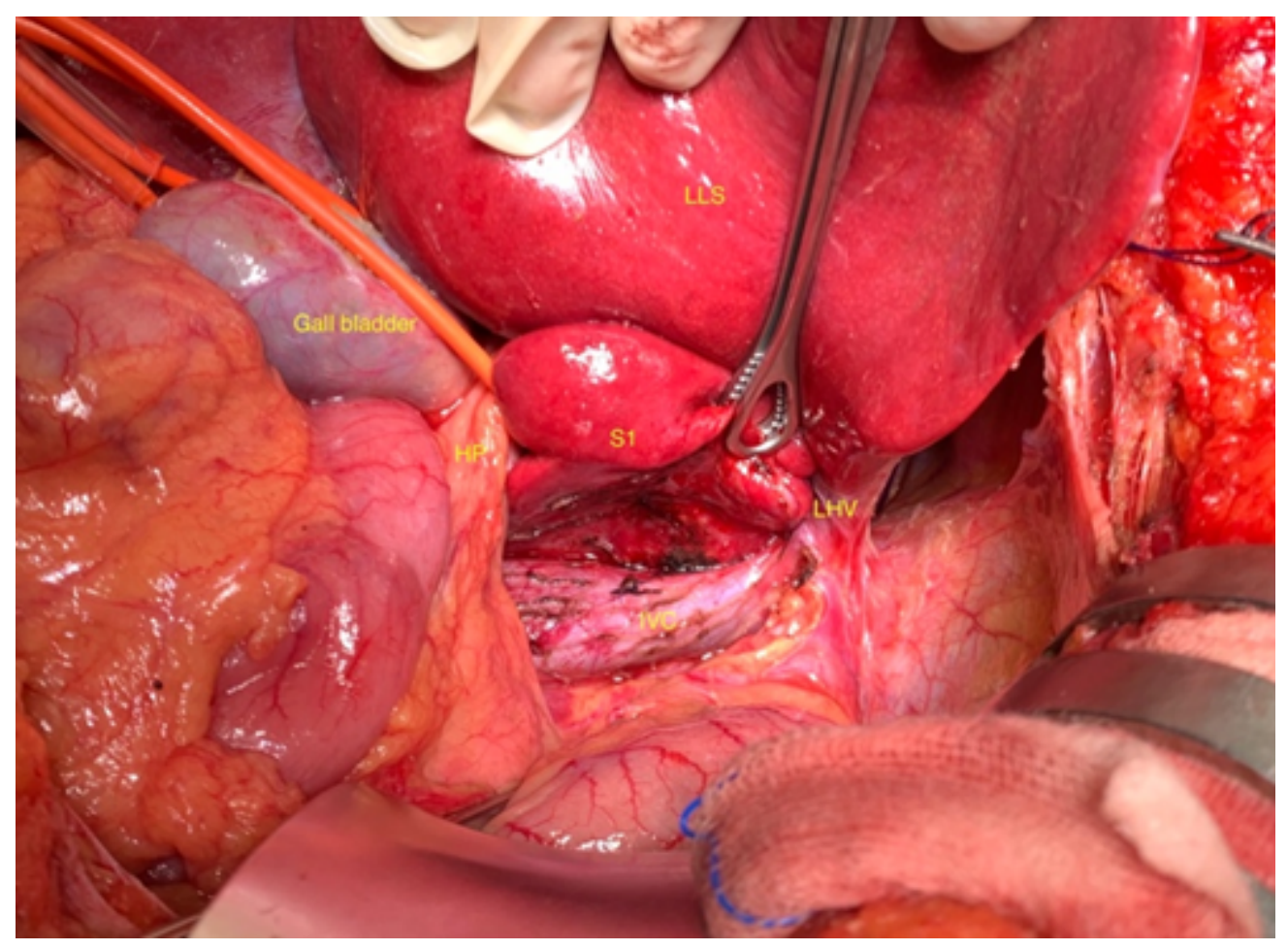

\section{Figure 1}

Ligation of the short hepatic veins from the subsegment I to the inferior vena cava (LLS: Left lateral section; LHV: left hepatic vein; IVC: Inferior vena cava; HP: Hepatic pedicle)

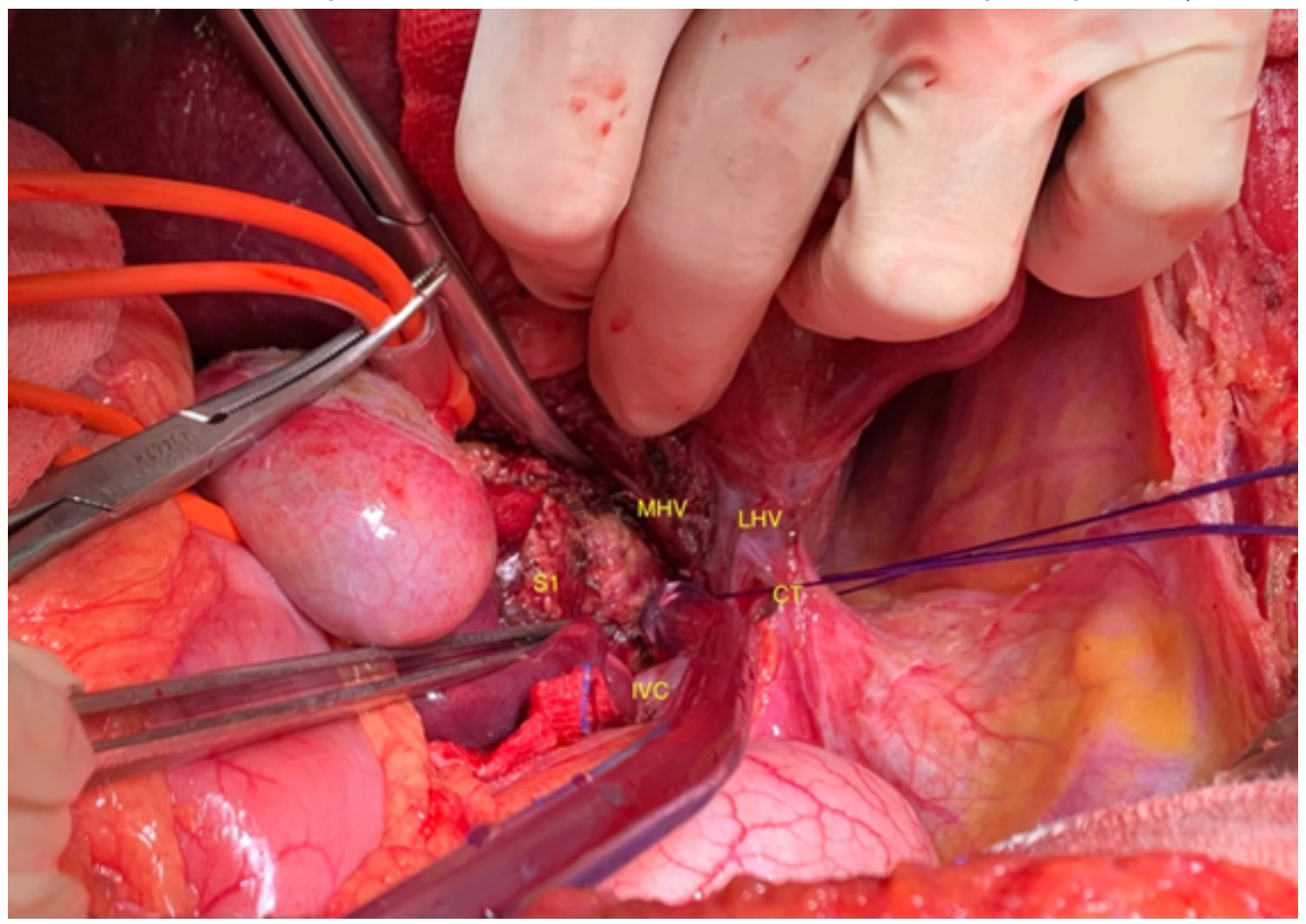

Figure 2 
The procedure usually starts from the left side, the liver parenchyma is resected just below the Arantius' ligament, separating the liver parenchyma from the inferior surface of the middle and left hepatic veins. At the bottom, we lift the right hepatic pedicle by using tapes, cut the caudate process and separate it from the right liver parenchyma (CT: common trunk; LHV: left hepatic vein; MHV: Medial hepatic vein)
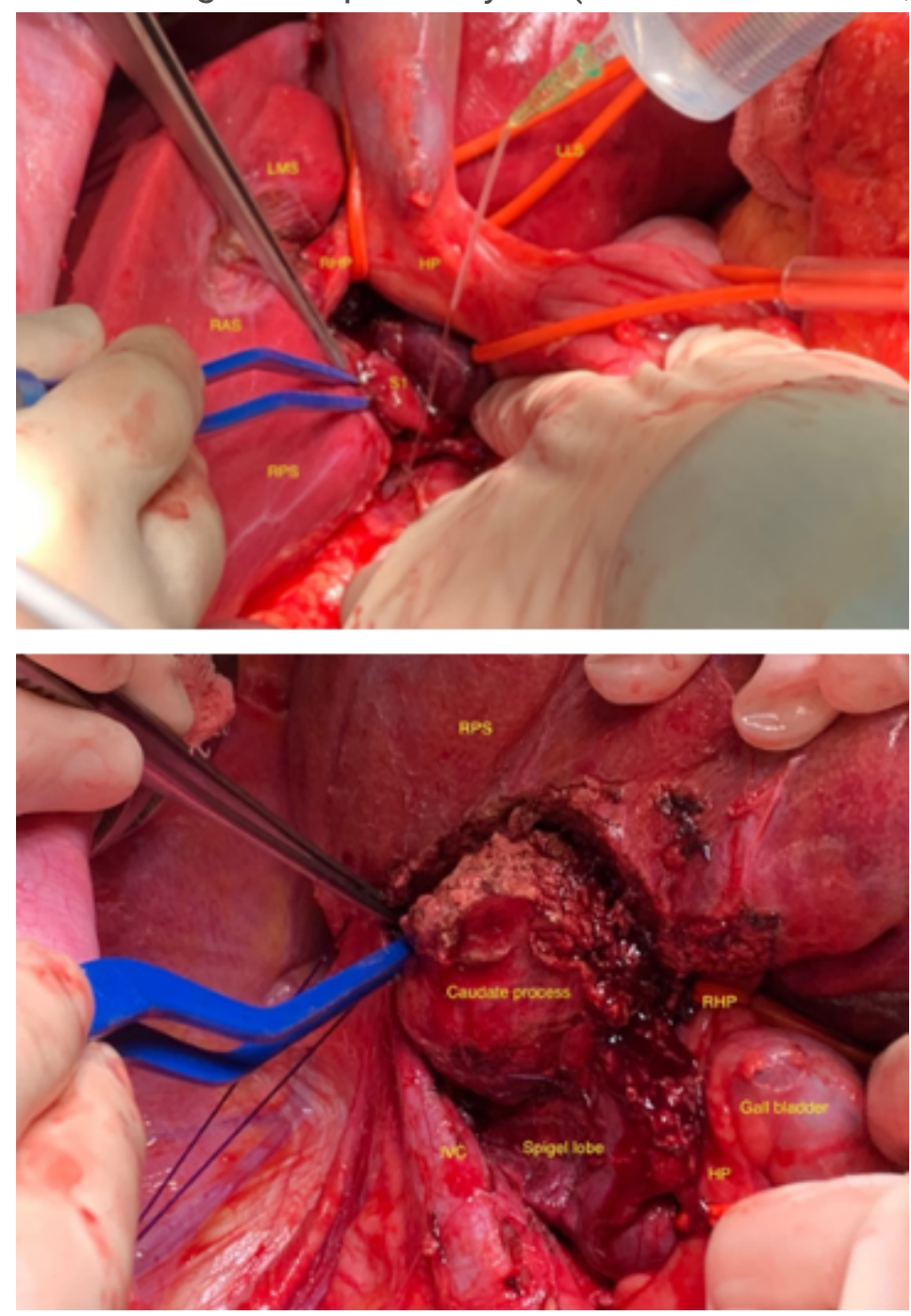

\section{Figure 3}

The liver parenchymal cutline from the right posterior approach after cutting the liver parenchyma from the left to the right border of the middle hepatic vein, the boundary is determined after ligation of the right hepatic pedicle of subsegment 1 (LLS: Left lateral section; LMS: left medial section; RAS: Right anterior section; RPS: Right posterior section; RHP: Right hepatic pedicle; HP: Hepatic pedicle) 


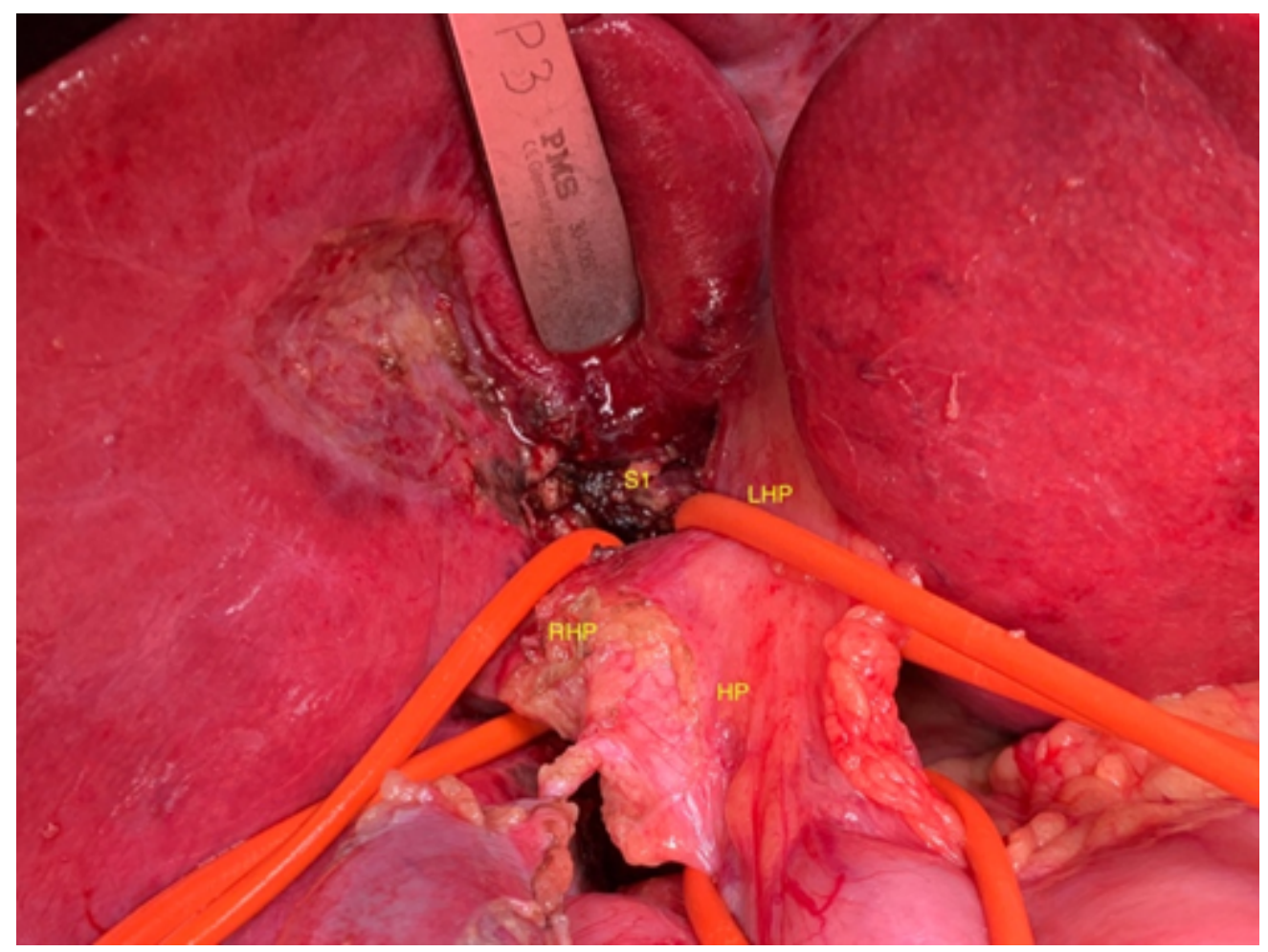

Figure 4

Right and left hepatic pedicles after controlling with tapes using Takasaki's technique (LHP: Left hepatic pedicle; RHP: Right hepatic pedicle; HP: Hepatic pedicle)
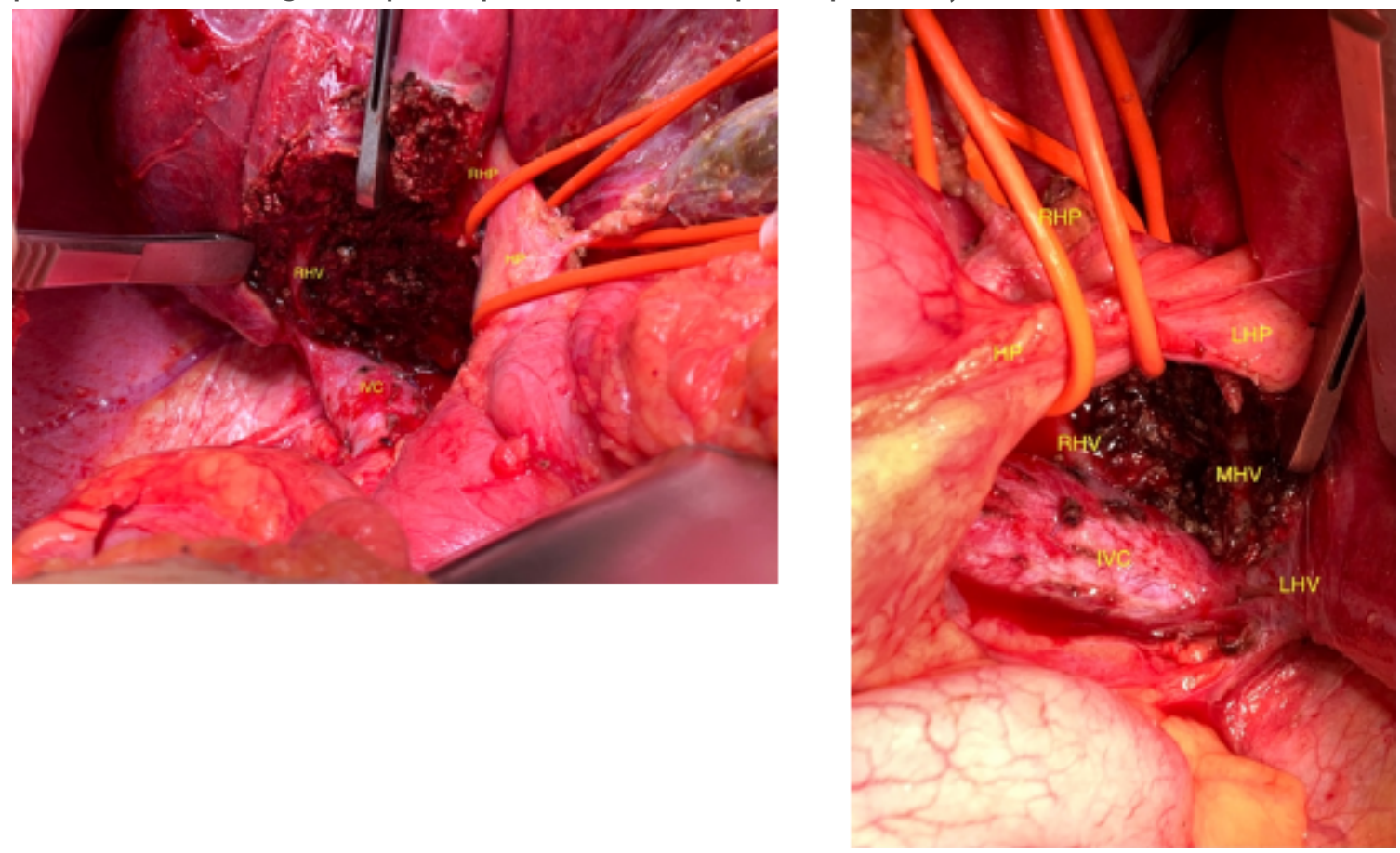

\section{Figure 5}

Resected liver parenchyma (LHV: left hepatic vein; MHV: medial hepatic vein; RHV: right hepatic vein; RHP: right hepatic pedicle; HP: hepatic pedicle; IVC: inferior vena cava) 


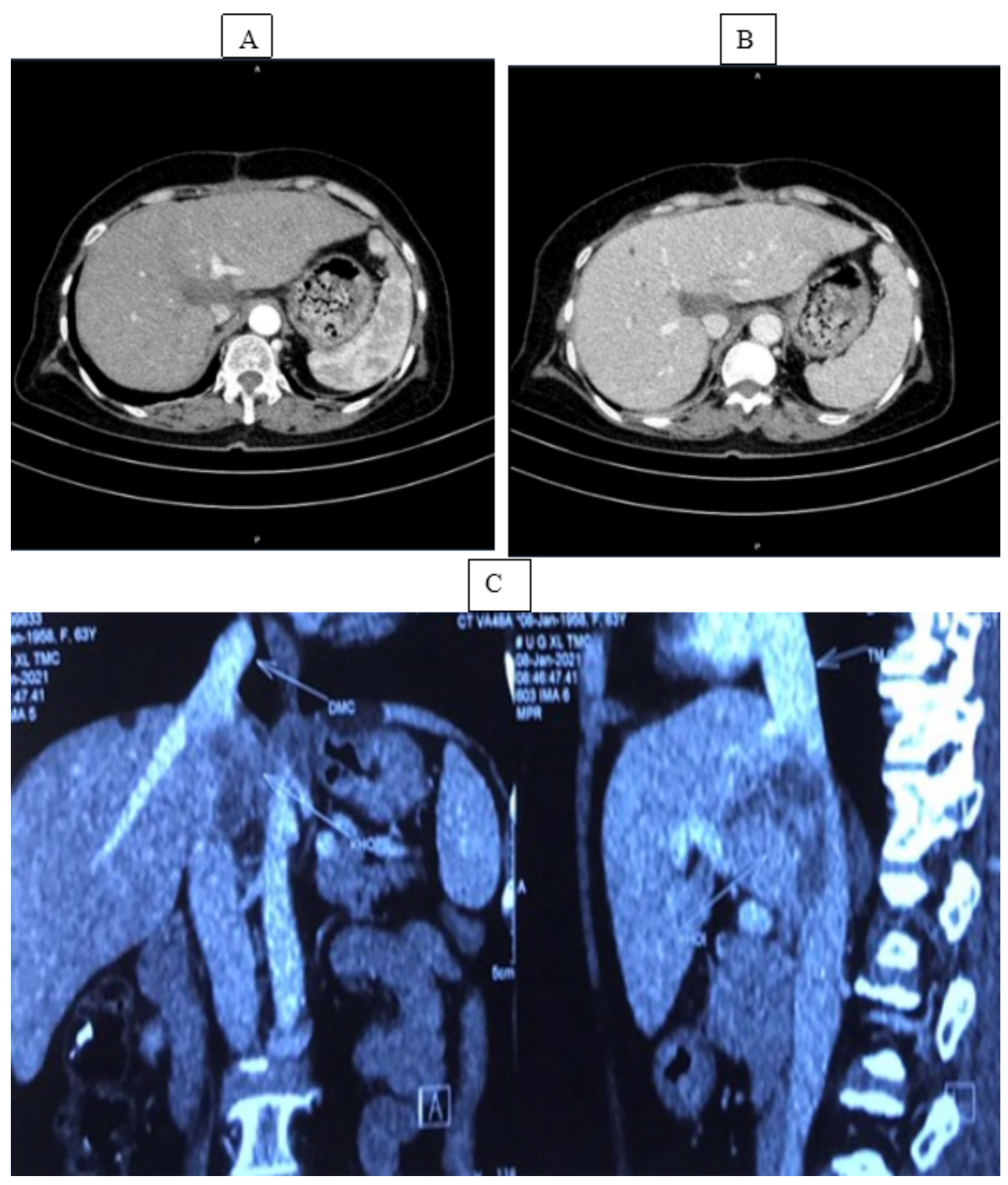

Figure 6

The CT result of the first patient showed a tumor occupying nearly the entire subsegment I with characteristics of HCC (A and B), at size $46 \mathrm{~mm}$, compressing inferior vena cava, close to the hepatic pedicle, right and left hepatic veins but not invasive, no thrombus portal vein (C) 


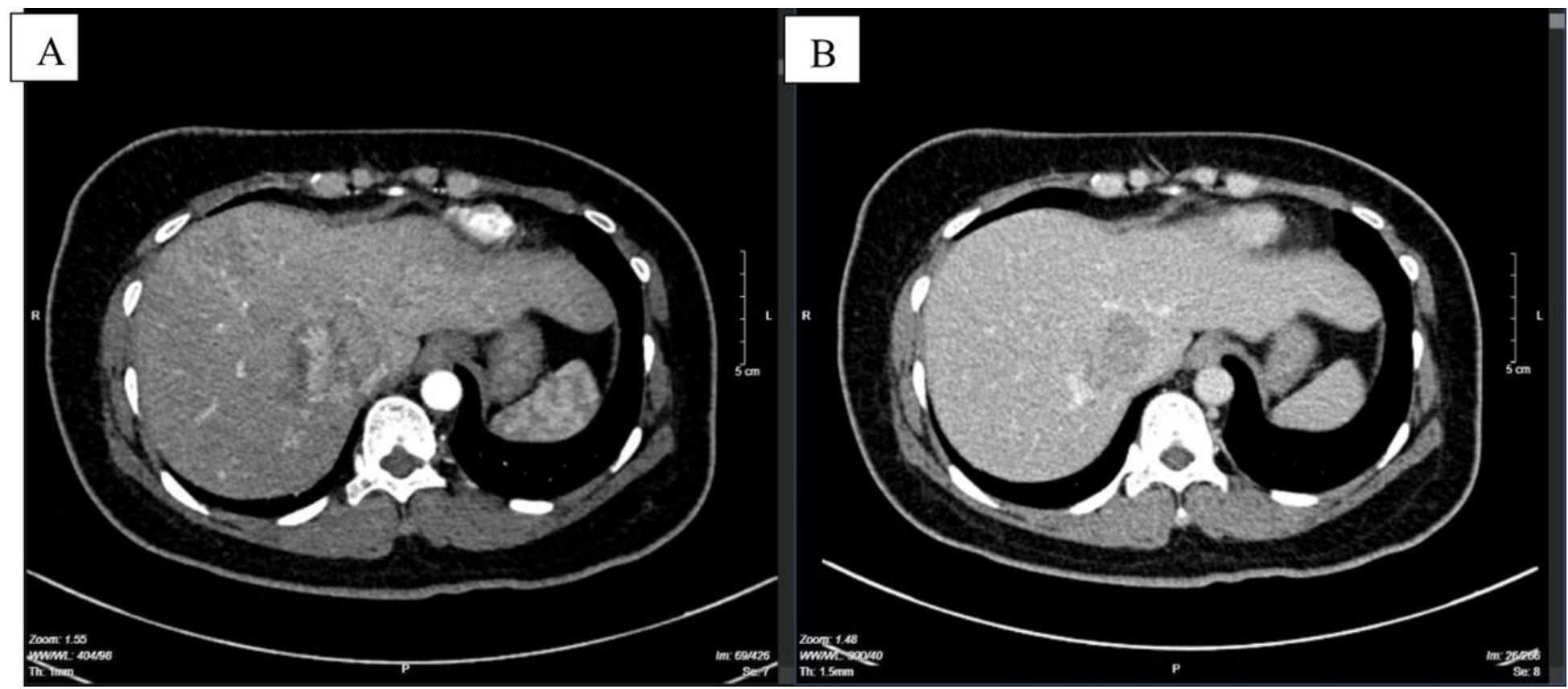

Figure 7

The CT result of the second patient showed a $45 \mathrm{~mm}$-size tumor of subsegment I, decreased density before injection, rapid drug enhancement in arterial phase (A), rapid drug removal in venous phase and irregular margins (B)

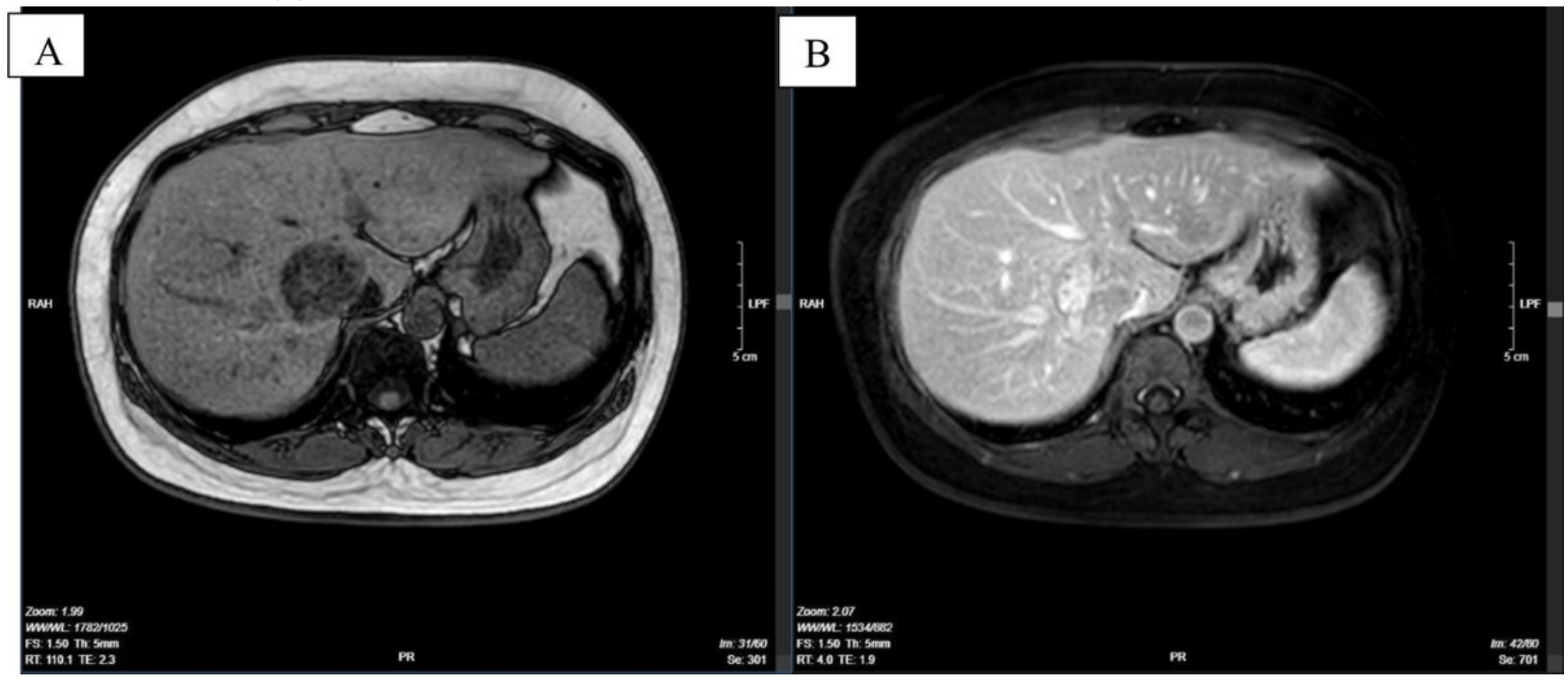

Figure 8

Liver MRI of the second patient showed a liver tumor about $5 \mathrm{~cm}$ in size, decreased signal on T1W out phase $(A)$, rapid enhancement in arterial phase and rapid drug elimination in venous phase, clearly limited but close to the hepatic veins and inferior vena cava (B). 